\title{
Comparison of Influenza Virus Detection Methods
}

\author{
Yusuke Takahara, ${ }^{1}$ Yuki Nakaya, ${ }^{1}$ Masato Yasuura, ${ }^{1}$ Hiroki Ashiba, ${ }^{1}$ \\ Penmetcha K. R. Kumar, ${ }^{2}$ and Makoto Fujimaki ${ }^{1 *}$ \\ ${ }^{1}$ Electronics and Photonics Research Institute, National Institute of Advanced Industrial \\ Science and Technology (AIST), Central 5, 1-1-1 Higashi, Tsukuba, Ibaraki 305-8565, Japan \\ ${ }^{2}$ Biomedical Research Institute, National Institute of Advanced Industrial \\ Science and Technology (AIST), Central 6, 1-1-1 Higashi, Tsukuba, Ibaraki 305-8566, Japan
}

(Received July 20, 2018; accepted September 5, 2018)

Keywords: biosensor, hemagglutination, plaque assay, immunoassay, polymerase chain reaction

In this study, we provide a cross-sectional comparison of existing influenza virus detection methods in terms of their sensitivity, sample volume required, and process time needed for an assay. Virological techniques, immunological techniques, and real-time polymerase chain reaction were examined. An identical lot of influenza virus stock was used for the experiments. The result gives an index for the evaluation of the performance abilities of virus detection systems.

\section{Introduction}

The fields of clinical medicine and preventive epidemiology call for simple and highperformance techniques for the sensitive detection and early identification of pathogens, such as the influenza virus. Influenza is an infectious disease that can be global in scope in annual outbreaks. Children ages 5 years or younger, the elderly aged 65 years or older, pregnant women, and patients with underlying diseases of the respiratory, metabolic, and immune systems are at heightened risk of increased severity of infection, according to the World Health Organization, with outbreaks profoundly affecting economic activities. ${ }^{(1)}$

The influenza virus is a negative-sense RNA virus of the Orthomyxoviridae family with an RNA genome of eight or seven segments. ${ }^{(2)}$ It is further classified into variable strains on the basis of the viral surface antigens hemagglutinin (HA) and neuraminidase (NA). The virus has been isolated from humans, other mammals, and birds, and the host range is determined by the combination of HAs and NAs. ${ }^{(2)}$ Eighteen HAs and 11 NAs have been identified so far. ${ }^{(3)}$ An antigenic shift occurs when different influenza virus strains recombine their genomes, resulting in a new strain having a mixture of HAs and NAs of original strains. ${ }^{(2)}$ This occasionally triggers outbreaks by producing a strain that has never infected humans. A well-known example is the 1968 influenza pandemic caused by the emergence of the H3HA subtype. ${ }^{(4)}$ Moreover, highly pathogenic viruses that have arisen from an avian influenza virus such as the H5N1 subtype have been threatening human public health. ${ }^{(2)}$ It is thus important that surveillance of the influenza virus is achieved precisely to prevent the pandemics.

${ }^{*}$ Corresponding author: e-mail: m-fujimaki@aist.go.jp https://doi.org/10.18494/SAM.2019.2062 
Methods of influenza virus detection include virological techniques, such as hemagglutination and plaque assays, ${ }^{(5)}$ immunological techniques, such as immunochromatography ${ }^{(6)}$ and enzymelinked immunosorbent assay (ELISA), ${ }^{(7)}$ and nucleic acid amplification techniques, such as realtime polymerase chain reaction (RT-PCR) ${ }^{(8)}$ and loop-mediated isothermal amplification. ${ }^{(9,10)}$ In addition, new methods have been developed on the basis of these detection strategies. ${ }^{(11,12)}$ However, to the best of our knowledge, there is no index for the comparison of the detection abilities of these methods. Such an index would be important for selecting an appropriate method matching for a purpose and evaluating the merits and demerits of a newly emerging technology.

In this research, we carried out a cross-sectional comparison of influenza virus detection methods to determine the index of sensitivity, sample volume required, and process time required for an assay using an identical lot of influenza virus stock.

\section{Materials and Methods}

\subsection{Virus}

Frozen aliquots of the influenza A virus strain A/Panama/2007/99 (H3N2), propagated from a purified plaque, were used in the experiments. In this study, dilution factor is used as an index for comparing sensitivity. Here, the dilution factor means the dilution ratio of the virus suspension used for each assay from the stock solution. Dilution with reagent solutions is not included in the calculation of the dilution factor in an assay in which reagents are mixed in a virus suspension. What is important is the original concentration of the sample, not the concentration in the final detection system, which depends on the volume of reagents added. If we compare the performance using the virus concentration after adding reagents, an assay taking into consideration the reagents added will result in the evaluation showing higher sensitivity, and the performance will be overestimated. This is the reason why the dilution factor as the index for comparing sensitivity does not include the dilution rate with reagent solutions.

\subsection{Hemagglutination assay}

Hemagglutination titers were determined by hemagglutination assay. The principle of the hemagglutination assay is shown in Fig. 1. Twofold serial dilutions of a virus stock were made in $25 \mu \mathrm{l}$ of phosphate-buffered saline (PBS) in 96-well U-bottom plates. Fifty microliters of $0.5 \%(\mathrm{v} / \mathrm{v})$ chicken red blood cells (Nippon Bio-test Laboratories, Japan) in PBS was added to each well. The plates were kept at $4{ }^{\circ} \mathrm{C}$ for $1 \mathrm{~h}$ and the hemagglutination patterns were read. Hemagglutination titers were determined from the last dilution showing complete hemagglutination. 


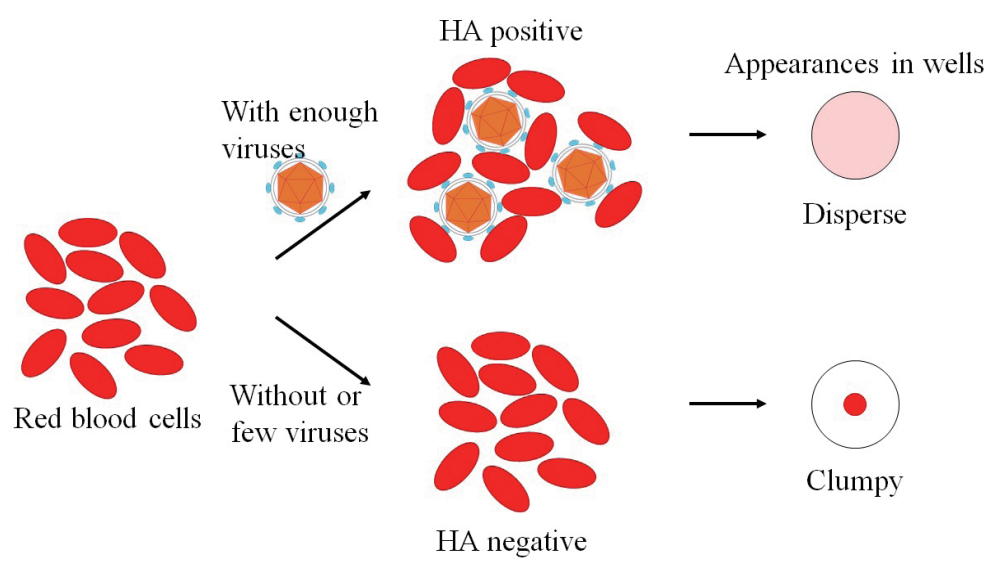

Fig. 1. (Color online) Schematic principle of the hemagglutination assay. Red blood cells mixed with virus particles containing HA are tightly bonded with each other, which turn into a distributed sheet in the entire well. Otherwise, free red blood cells gather at the bottom of the well.

\subsection{Plaque assay}

The infectivity of the virus was determined by plaque assay. ${ }^{(5)}$ The principle of the plaque assay is shown in Fig. 2. The virus was serially diluted 10-fold in Dulbecco's modified Eagle's medium (Thermo Fisher Scientific, USA) supplemented with 10\% fetal bovine serum, 100 unit/ml penicillin, and $100 \mu \mathrm{g} / \mathrm{ml}$ streptomycin (Thermo Fisher Scientific). Madin-Darby canine kidney cell monolayers maintained in 6-well tissue culture plates were inoculated with $400 \mu \mathrm{l}$ of the virus dilutions and incubated at $37{ }^{\circ} \mathrm{C}$ for $1 \mathrm{~h}$ with rocking every $15 \mathrm{~min}$. The inoculum was removed and $2 \mathrm{ml}$ of serum-free medium (KBM220, Kohjin Bio, Japan) containing $0.8 \%$ SeaPlaque agarose (50101; Lonza, USA) and $1.25 \mu \mathrm{g} / \mathrm{ml}$ acetylated trypsin (Sigma-Aldrich, USA) was added to each well to be solidified. The plates were incubated for 3 days at $37{ }^{\circ} \mathrm{C}$ in $5 \% \mathrm{CO}_{2}$ and plaques were counted. Infectivity was calculated as plaque forming units (pfu) per ml. Assays were performed in three independent experiments.

\subsection{Chromatographic rapid diagnostic test (RDT)}

The ESPLINE Influenza A \& B-N (Fujirebio, Japan) chromatographic influenza RDT was used. This test is currently commercially available in Japan. The sensitivity of the kit to another influenza virus isolate was previously reported. ${ }^{(13,14)}$ The detection principle is based on an antigen-antibody reaction with monoclonal antibody(ies) specific to nucleoprotein (NP), which is one of the most abundant proteins in influenza virions. Twenty microliters of each 10-fold serial virus dilution in PBS was mixed with $200 \mu 1$ of sample preparation buffer. Twenty microliters of the mixtures was subsequently dispensed on the immunoassay cassettes. Results were interpreted on the basis of observations with the naked eye after 15 min incubation at room temperature. This assay was repeated independently three times. 



Fig. 2. (Color online) Schematic principle of the plaque assay. Infected cells are dead and observed as plaques (white circles) in the assay. Each plaque is considered to be derived from a single cell infected by the virus. Virus titers are calculated as $\mathrm{pfu} / \mathrm{ml}$, which is based on the plaque number obtained and its dilution factor. For example, if you see 7 plaques in a monolayer inoculated with $1 \mathrm{ml}$ of $10^{3}$-fold diluted virus, the pfu is calculated as $7 \times 10^{3} \mathrm{pfu} / \mathrm{ml}$.

\subsection{Sandwich ELISA}

A commercially available sandwich ELISA kit (Influenza A virus Nucleoprotein Antigen ELISA Kit, DEIA-CL036; Creative Diagnostics, USA) was used for NP antigen detection in accordance with the manufacturer's instruction. Briefly, the virus was serially diluted 10-fold in PBS and $200 \mu \mathrm{l}$ of each dilution was mixed with $50 \mu \mathrm{l}$ of the sample preparation reagent. One hundred microliters of each mixture including $80 \mu \mathrm{l}$ of virus dilution was added to the antibody-coated plate and incubated for $30 \mathrm{~min}$ at room temperature. The influenza A virus detection antibody was dispensed with an equal volume of a sample mixture and incubated for $45 \mathrm{~min}$. After washing the plate six times with the wash buffer, the sample was reacted with $100 \mu \mathrm{l}$ of chromogen for $10 \mathrm{~min}$ for color development, and the reaction was stopped by adding $100 \mu \mathrm{l}$ of stop solution. The optical density (OD) at $450 \mathrm{~nm}$ was measured using an iMark microplate reader (Bio-Rad, USA). The cutoff value was determined by multiplying the negative control value by 2 , where the negative control was provided with the kit. Assays were carried out in triplicate independently.

\subsection{One-step RT-PCR}

One-step RT-PCR was employed to detect influenza virus RNA. Total RNA was prepared from $140 \mu$ l of the virus stock of serially diluted 10-fold in PBS using a QIAamp ${ }^{\circledR}$ Viral RNA Mini Kit (Qiagen, USA) in accordance with the manufacturer's instructions. Total RNA was eluted with $60 \mu \mathrm{l}$ of buffer AVE included in the kit. RT-PCR was performed using a QuantiTect ${ }^{\circledR}$ Probe RT-PCR Kit (Qiagen) in accordance with the manufacturer's instructions. The PCR mixture consisted of $10 \mu 1$ of $2 \times$ QuantiTect Probe PCR Master Mix, $0.2 \mu 1$ of QuantiTect RT Mix, $0.8 \mu \mathrm{l}$ of $10 \mu \mathrm{M}$ forward primer, $0.8 \mu \mathrm{l}$ of $10 \mu \mathrm{M}$ reverse primer, $0.4 \mu \mathrm{l}$ of $5 \mu \mathrm{M}$ probe, $3.8 \mu \mathrm{l}$ of water, and $4 \mu \mathrm{l}$ of the RNA template, which is equivalent to $9.3 \mu 1$ of virus dilutions. Thermal cycling conditions and the primer design were described previously. ${ }^{\left({ }^{(8)}\right.}$ Thermal cycling was performed as follows: $30 \mathrm{~min}$ at $50{ }^{\circ} \mathrm{C}$ to activate reverse transcriptase, followed by initial denaturation for $15 \mathrm{~min}$ at $95{ }^{\circ} \mathrm{C}$ with subsequent 45 cycles of amplification (denaturation at $94{ }^{\circ} \mathrm{C}$ for $15 \mathrm{~s}$ and annealing as well as an extension at $60{ }^{\circ} \mathrm{C}$ for $60 \mathrm{~s}$ ) using 
MyGo Mini (IT-IS Life Science Ltd., Ireland). The following probe and primers were used in the experiment: MP-96-75ProbeAs (5'-FAM-ATYTCGGCTTTGAGGGGGCCTG-MGB-3'), MP-39-67For (5'-CCMAGGTCGAAACGTAYGTTCTCTCTATC-3'), and MP-183-153Rev (5'-TGACAGRATYGGTCTTGTCTTTAGCCAYTCCA-3'). The assay was performed in triplicate.

\section{Results}

\subsection{Stock virus characteristics}

To determine the stock virus characteristics used in this study, we performed two classical virological assays: the hemagglutination and plaque assays. These two assays revealed that the hemagglutination titer of the virus stock was 256 (Fig. 3) and the concentration of infectious virus particles was $4.03 \times 10^{7} \mathrm{pfu} / \mathrm{ml}$. The amount of the virus suspension used in the hemagglutination assay was $25 \mu \mathrm{l}$ per well and the time required to detect the virus (observing aggregation) was $\sim 2 \mathrm{~h}$, which included $1 \mathrm{~h}$ of virus incubation. As the hemagglutination titer was 256 , the detection limit (the detectable highest dilution factor) was between $10^{2}$ and $10^{3}$. In the plaque assay, the amount of the virus suspension per assay was $400 \mu 1$. The plaque assay detects infectious virus particles. Since the virus stock concentration was $4.03 \times 10^{7} \mathrm{pfu} / \mathrm{ml}$, approximately 16 infectious virus particles were contained in $400 \mu 1$ of the sample diluted $10^{6}$ fold. The detectable highest dilution factor of the plaque assay was about $10^{7}$.

\subsection{Immunological detection assays based on antigen-antibody interaction}

Figure 4 shows a photograph of the virus detection result obtained using the RDT, ESPLINE Influenza A \& B-N. A positive band was observed on the test line in the testing of samples diluted 10 - and $10^{2}$-fold. In contrast, it was difficult to discern positive bands in samples diluted $10^{3}$-fold or greater. This result means that the detectable highest dilution factor of the RDT kit was between $10^{2}$ and $10^{3}$.

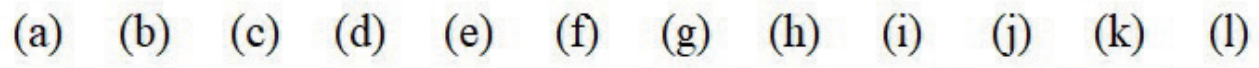

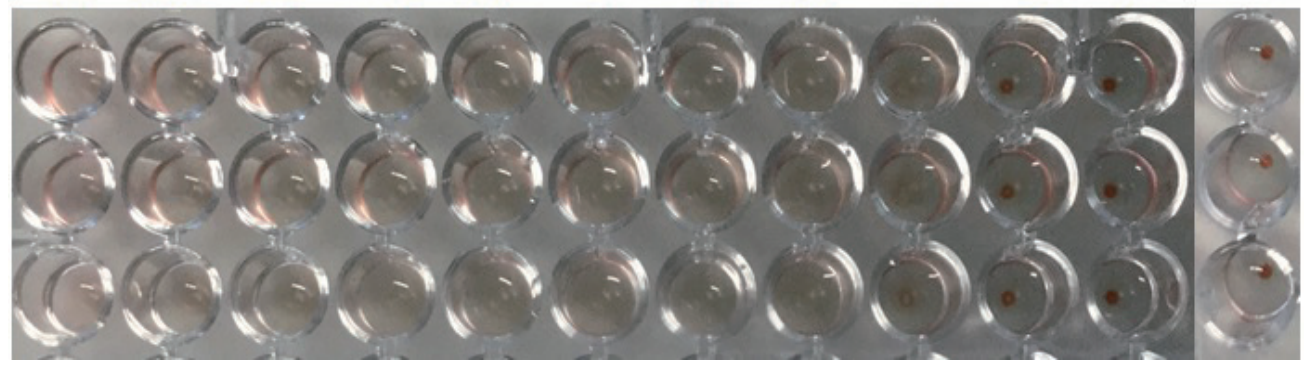

Fig. 3. (Color online) Titration of the virus stock by the hemagglutination assay. The dilution factors of the virus stock used in the assay (a), (b), (c), (d), (e), (f), (g), (h), (i), (j), and (k) were 1, 2, $2^{2}, 2^{3}, 2^{4}, 2^{5}, 2^{6}, 2^{7}, 2^{8}, 2^{9}$, and $2^{10}$, respectively. Negative control (PBS) was applied instead of the virus (l). 


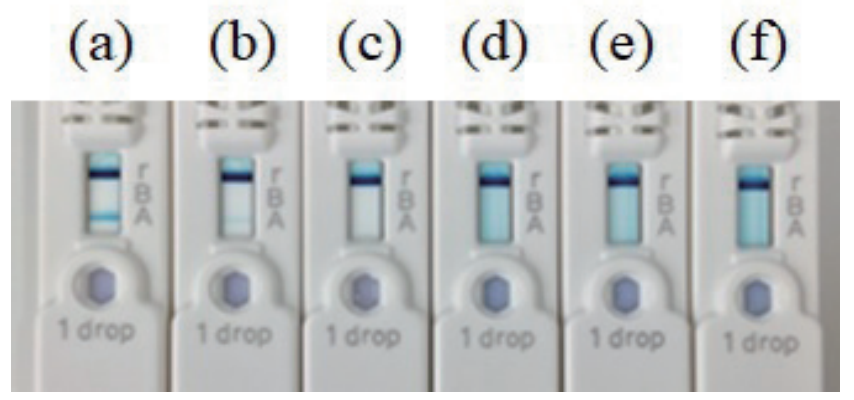

Fig. 4. (Color online) Photograph of the virus detection result obtained using the RDT, ESPLINE Influenza A \& B-N. The dilution factors of the virus stock applied to the cassettes (a), (b), (c), (d), and (e) were 10, $10^{2}, 10^{3}, 10^{4}$, and $10^{5}$, respectively. Negative control (PBS) was applied to the cassette (f).

Figure 5 shows the result of the NP antigen detection using the commercially available ELISA kit. Each data point expresses the average of three independent experiments. Each error bar indicates \pm 3 standard deviations. The cutoff value was determined in accordance with the manufacturer's instruction using the negative control provided with the kit. The detectable highest dilution factor of the NP capture ELISA was between $10^{3}$ and $10^{4}$. The total incubation time of the NP capture ELISA was $85 \mathrm{~min}$, and all the assays took approximately $2-3 \mathrm{~h}$.

\subsection{Real-time RT-PCR assays}

Figure 6 depicts cycle threshold $(\mathrm{Ct})$ values against the original virus stock dilution factor in the RT-PCR analysis using the MyGo Mini. Each value expresses the average of triplicate data and each error bar indicates the standard deviation. The detectable highest dilution factor of the method was $10^{7}-10^{8}$. The PCR analysis took approximately $3 \mathrm{~h}$ for the detection, which included 30 min for viral RNA extraction and 100 min for amplification.

\section{Discussion}

Table 1 summarizes the detection results. In the table, 'target' indicates the substances detected by each method. 'Sample volume' is the amount of the virus sample used for the sample preparation at the beginning of each assay, which means that the volumes of reagents used in the assays are excluded from the calculation. The numbers in parentheses are the amount of virus samples actually introduced into the assays. 'Process time' is the time required for virus detection by each method. The number in parentheses for the hemagglutination assay and ELISA indicates the time required for the incubation processes, and that for the RT-PCR indicates the time required for the amplification. 'Normalized sensitivity' gives an exponent of the detectable highest dilution factor of each assay normalized by the value of the hemagglutination assay.

The virological virus titration methods included the hemagglutination and plaque assays. The hemagglutination assay is widely used for the quantitative evaluation of influenza virus. As shown in Table 1, the hemagglutination assay displayed a low sensitivity and was not suitable for the detection of a trace amount of the virus. However, since chicken red blood 


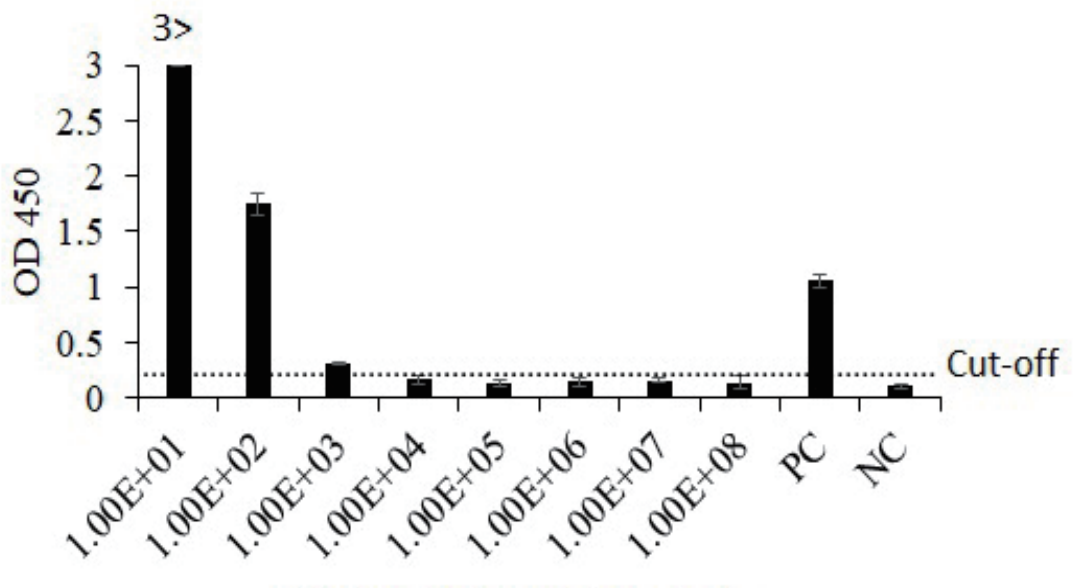

Dilution factor of virus stock

Fig. 5. Result of the NP antigen detection using the commercially available ELISA kit. 'PC' and 'NC' indicate the positive and negative controls included in the kit, respectively.

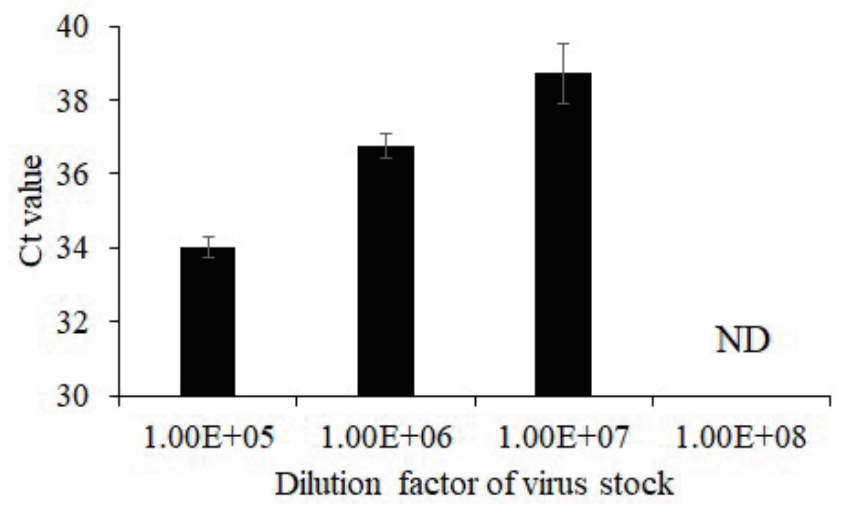

Fig. 6. Ct values against the dilution factor of the virus stock observed in the RT-PCR analysis. 'ND' indicates that substantial amplification was not observed.

Table 1

Summary of results of comparison of the influenza virus detection methods.

\begin{tabular}{|c|c|c|c|c|c|}
\hline & Target & $\begin{array}{l}\text { Sample volume } \\
(\mu \mathrm{l})\end{array}$ & Process time & $\begin{array}{l}\text { Detectable highest } \\
\text { dilution factor }\end{array}$ & $\begin{array}{l}\text { Normalized } \\
\text { sensitivity }\end{array}$ \\
\hline Hemagglutination assay & Virus particles & 25 & $2 \mathrm{~h}(1 \mathrm{~h})$ & $10^{2}-10^{3}$ & 0 \\
\hline Plaque assay & Infectious particles & 400 & 3 days & $\sim 10^{7}$ & 4 \\
\hline Chromatographic RDTs & Protein (NP) & $20(1.8)$ & $15 \mathrm{~min}$ & $10^{2}-10^{3}$ & 0 \\
\hline ELISA & Protein (NP) & $200(80)$ & $2-3 \mathrm{~h}(85 \mathrm{~min})$ & $10^{3}-10^{4}$ & 1 \\
\hline RT-PCR & RNA (M1 gene) & $140(9.3)$ & $3 \mathrm{~h}(100 \mathrm{~min})$ & $10^{7}-10^{8}$ & 5 \\
\hline
\end{tabular}

cells react with all the viral surface antigens and the measurement result reflects the number of viral particles in the sample and can be obtained without the need of a special device or a measurement kit, the hemagglutination assay is considered to be suitable for use as a standard of the sensitivity of immunoassay or PCR, which evaluates protein or RNA originating from virus particles. 
We determined the stock virus infectious titer as $4.03 \times 10^{7} \mathrm{pfu} / \mathrm{ml}$ by the plaque assay. Among the assays we assessed, only the plaque method targets infectious viruses. The other approaches cannot determine virus infectivity. The plaque assay is highly sensitive and can detect only infectious viruses, so it is possible to evaluate the number of viruses that may harm humans. However, since it takes days to obtain the results, it is not suitable as a virus sensor.

The RDT provided detection in 15 min from a $20 \mu \mathrm{l}$ sample. The highest dilution for detection was $10^{2}-10^{3}$. In the case of using the commercially available ELISA kit targeting NP, a sensitivity one digit higher than that of the RDT was obtained with a measurement time of 2-3 h. The measurement time required for the RDT is short and the assay method is simple, but its low sensitivity is a problem. The ELISA kit is highly sensitive, highly quantifiable, and excellent as a virus concentration evaluation tool. However, the time required for measurement is long, and the technical level of the measurer has a large effect on the measurement result.

The detection sensitivity of immunosensors based on the antigen-antibody reaction is sometimes expressed as pfu, as compared with the detection results obtained by the plaque assay. However, as described above, since the plaque assay is a tool for evaluating the number of infectious virus particles and the immunosensors are used for evaluating the concentration of the target protein, it is not appropriate to evaluate the sensitivity of the immunosensors using pfu. If the sensitivity of the immunosensor is to be evaluated, it is considered better to compare it with hemagglutination titer, which reflects the virus particle number.

In the RT-PCR using a conventional thermal cycler, a $140 \mu 1$ specimen volume was applied. The amplification step took $100 \mathrm{~min}$ and the highest dilution for detection was $10^{7}-10^{8}$. Recently, it has been reported that a microfluidic RT-PCR device yielded a similar sensitivity to the conventional thermal cycler RT-PCR device, where the time required for the amplification was approximately one-tenth of that of the conventional thermal cycler RT-PCR. ${ }^{(15,16)}$ The sensitivity of the RT-PCR is higher than that of the plaque assay, because the RT-PCR detects RNA not only in infectious viruses but also in noninfectious ones. The sensitivity of the RTPCR is considered to be sufficient to detect viruses in the environment and the RT-PCR is applied as a sensor for the prevention of infections. On the other hand, viruses that are likely to infect humans are only infectious virus particles. The detection of even trace amounts of viral RNA or viral proteins is no guarantee of a danger of infection due to an infectious virus. Therefore, for preventing infection, a method that can enumerate infectious viruses, such as the plaque assay, is suitable. Several techniques for evaluating virus infectivity based on PCR have been reported. ${ }^{(17-19)}$ In the future, it will be necessary to develop infectious virus sensors that can replace the plaque assay.

In this study, we compared various virus detection methods with different detection principles and targets. As mentioned above, the hemagglutination assay, which provides a value that correlates with the number of virus particles, can be used as an evaluation standard of immunoassays and RT-PCR that detect viral proteins and viral RNAs, respectively. However, depending on the collection method and/or preservation state of the virus, the virus particles or RNA may be damaged, and the protein may be degraded. If mutation occurs in the RNA targeted by PCR or the RNA is not present, the virus can be detected by immunoassay, but not by PCR. Thus, the values obtained by these methods do not necessarily correlate with each other. 


\section{Conclusions}

This is the first study directly comparing the volumes, times, and sensitivities between different assays using an identical virus stock. These parameters significantly varied as expected. Although smaller amount, shorter time, higher sensitivity, and accuracy are desired and pursued in an assay, it is obvious that every method has both merits and demerits in different points. This clearly indicates that we need to change the methods of testing the influenza virus depending on the situation. We hope that this study will be a helpful for choosing a virus detection method in future examinations.

\section{References}

1 World Health Organization, Influenza (Seasonal): https://www.who.int/en/news-room/fact-sheets/detail/ influenza-(seasonal) (accessed December 2018).

2 B. Arbeitskreis and Untergruppe «Bewertung Blutassoziierter Krankheitserreger»: Transfus. Med. Hemother. 36 (2009) 32. https://doi.org/10.1159/000197314

3 S. Tong, X. Zhu, Y. Li, M. Shi, J. Zhang, M. Bourgeois, H. Yang, X. Chen, S. Recuenco, J. Gomez, L. M. Chen, A. Johnson, Y. Tao, C. Dreyfus, W. Yu, R. McBride, P. J. Carney, A. T. Gilbert, J. Chang, Z. Guo, C. T. Davis, J. C. Paulson, J. Stevens, C. E. Rupprecht, E. C. Holmes, I. A. Wilson, and R. O. Donis: PLoS Pathog. 9 (2013) e1003657-1. https://doi.org/10.1371/journal.ppat.1003657

4 Y.-C. Hsieh, T.-Z. Wu, D.-P. Liu, P.-L. Shao, L.-Y. Chang, C.-Y. Lu, C.-Y. Lee, F.-Y. Huang, and L.-M. Huang: J. Formos. Med. Assoc. 105 (2006) 1. https://doi.org/10.1016/S0929-6646(09)60102-9

5 J. Xue, B. S. Chambers, S. E. Hensley, and C. B. López: Front. Microbiol. 7 (2016) 326-1. https://doi. org/10.3389/fmicb.2016.00326

6 L. T. Nguyen, K. Nakaishi, K. Motojima, A. Ohkawara, E. Minato, J. Maruyama, T. Hiono, K. Matsuno, M. Okamatsu, T. Kimura, A. Takada, H. Kida, and Y. Sakoda: PLoS One 12 (2017) e0182228. https://doi. org/10.1371/journal.pone.0182228

7 C. M. Oshansky, S. Wong, T. Jeevan, H. S. Smallwood, R. J. Webby, S. C. Shafir, and P. G. Thomas: mBio 5 (2014) e02107-14. https://doi.org/10.1128/mBio.02107-14

8 M. Nakauchi, Y. Yasui, T. Miyoshi, H. Minagawa, T. Tanaka, M. Tashiro, and T. Kageyama: J. Virol. Meth. 171 (2011) 156. https://doi.org/10.1016/j.jviromet.2010.10.018

9 T. Notomi, H. Okayama, H. Masubuchi, T. Yonekawa, K. Watanabe, N. Amino, and T. Hase: Nucleic Acids Res. 28 (2000) e63. https://doi.org/10.1093/nar/28.12.e63

10 L. L. M. Poon, C. S. W. Leung, K. H. Chan, J. H. C. Lee, K. Y. Yuen, Y. Guan, and J. S. M. Peiris1: J. Clin. Microbiol. 43 (2005) 427. http://doi.org/10.1128/JCM.43.1.427-430.2005

11 T. Ono, T. Oe, Y. Kanai, T. Ikuta, Y. Ohno, K. Maehashi, K. Inoue, Y. Watanabe, S. Nakakita, Y. Suzuki, T. Kawahara, and K. Matsumoto: Jpn. J. Appl. Phys. 56 (2017) 030302. https://doi.org/10.7567/JJAP.56.030302

12 K. Nomura, S. C. B. Gopinath, T. Lakshmipriya, N. Fukuda, X. Wang, and M. Fujimaki: Nat. Commun. 4:2855 (2013). https://doi.org/10.1038/ncomms3855

13 Y. Sakai-Tagawa, M. Ozawa, D. Tamura, M. T. Le, C. A. Nidom, N. Sugaya, and Y. Kawaoka: J. Clin. Microbiol. 48 (2010) 2872. https://doi.org/10.1128/JCM.00439-10

14 Y. Sakai-Tagawa, M. Ozawa, S. Yamada, Y. Uchida, T. Saito, K. Takahashi, N. Sugaya, M. Tashiro, and Y. Kawaoka: Microbiol. Immunol. 58 (2014) 600. https://doi.org/10.1111/1348-0421.12185

15 S. Furutani, Y. Hagihara, and H. Nagai: Meat Sci. 131 (2017) 56. https://doi.org/10.1016/j.meatsci.2017.04.009

16 S. Furutani, N. Naruishi, Y. Hagihara, and H. Nagai: Anal. Bioanal. Chem. 408 (2016) 5641. https://doi. org/10.1007/s00216-016-9668-8

17 S. Nuanualsuwan and D. O. Cliver: J. Virol. Methods 104 (2002) 217. https://doi.org/10.1016/S01660934(02)00089-7

18 J. R. Toppinga, H. Schnerr, J. Haines, M. Scott, M. J. Carter, M. M. Willcocks, K. Bellamy, D. W. Brown, J. J. Gray, C. I. Gallimore, and A. I. Knight: J. Virol. Methods 156 (2009) 89. https://doi.org/10.1016/ j.jviromet.2008.10.024

19 S. Parshionikar, I. Laseke, and G. S. Fout: Appl. Environ. Microbiol. 76 (2010) 4318. https://doi.org/10.1128/ AEM.02800-09 